\title{
Dimerization transitions in spin-1 chains
}

\author{
Natalia Chepiga, ${ }^{1}$ Ian Affleck, ${ }^{2}$ and Frédéric Mila ${ }^{1}$ \\ ${ }^{1}$ Institute of Physics, Ecole Polytechnique Fédérale de Lausanne (EPFL), CH-1015 Lausanne, Switzerland \\ ${ }^{2}$ Department of Physics and Astronomy, University of British Columbia, Vancouver, British Columbia, Canada V6T $1 Z 1$
}

(Received 4 March 2016; published 20 June 2016)

\begin{abstract}
We study spontaneous dimerization transitions in a Heisenberg spin-1 chain with additional next-nearestneighbor and three-site interactions using extensive numerical simulations and a conformal field-theory analysis. We show that the transition can be second order in the Wess-Zumino-Witten (WZW) SU(2) 2 or Ising universality class, or first order. We argue that these features are generic because of a marginal operator in the WZW SU(2) 2 model and because of two topologically distinct nondimerized phases with or without edge states. We also provide explicit numerical evidence of conformal towers of singlets inside the spin gap at the Ising transition. Implications for other models are briefly discussed.
\end{abstract}

DOI: 10.1103/PhysRevB.93.241108

Topological matter is currently attracting a lot of attention. One of the first examples is the spin-1 Heisenberg chain, which has a bulk gap [1] but spin-1/2 edge states [2,3]. Spin-1 chains with more general interactions have been extensively studied over the years, and they have in particular been shown to undergo a spontaneous dimerization in the presence of a negative biquadratic interaction at an integrable critical point [4,5]. The universality class of this critical point is $\mathrm{SU}(2)_{2}$ Wess-Zumino-Witten (WZW) with central charge $c=3 / 2$ [6-8]. It has been identified in other models exhibiting spontaneous dimerization [9], and it is usually considered to describe the generic behavior of spin- 1 chains at the transition to a spontaneously dimerized phase.

In this Rapid Communication, we identify two other generic possibilities, Ising and first order, and we show that these alternatives are natural consequences of general properties: (i) the presence of topological and nontopological phases with and without edge states, respectively, and (ii) the existence of a marginal operator in the WZW SU(2) 2 model. We also show that combining density-matrix renormalization group (DMRG) [10-13] simulations with conformal field-theory (CFT) predictions for open systems gives access to the conformal towers of the critical lines, including that of singlets inside the spin gap on the Ising line.

We consider the spin-1 chain Hamiltonian,

$$
\begin{aligned}
H= & \sum_{i}\left(J_{1} \mathbf{S}_{i} \cdot \mathbf{S}_{i+1}+J_{2} \mathbf{S}_{i-1} \cdot \mathbf{S}_{i+1}\right) \\
& +\sum_{i} J_{3}\left[\left(\mathbf{S}_{i-1} \cdot \mathbf{S}_{i}\right)\left(\mathbf{S}_{i} \cdot \mathbf{S}_{i+1}\right)+\text { H.c. }\right] .
\end{aligned}
$$

On top of the standard Heisenberg coupling $J_{1}$, it includes two of the three interactions that appear in next-to-leading order in the strong-coupling expansion of the two-band Hubbard model: the next-nearest-neighbor (NNN) interaction $J_{2}$ and a three-site interaction with coupling strength $J_{3}$. The biquadratic interaction $\left(\mathbf{S}_{i} \cdot \mathbf{S}_{i+1}\right)^{2}$ has been omitted for simplicity. We set $J_{1}=1$ throughout the Rapid Communication and concentrate on the case of $J_{2}, J_{3} \geqslant 0$.

Let us first summarize the main results obtained using extensive DMRG simulations and exact diagonalizations. The phase diagram as a function of $J_{2}$ and $J_{3}$ consists of three phases, each of which may be schematically illustrated by a diagram with lines indicating valence-bond singlets formed between pairs of sites (see Fig. 1): a Haldane phase with one valence bond per $J_{1}$ bond, a NNN-Haldane phase with one valence bond per $J_{2}$ bond, and a dimerized phase with two valence bonds on every other $J_{1}$ bond. The characterization of the short-range correlations (including disorder and Lifshitz lines) will be reported elsewhere [14].

The transition between the Haldane and the NNN-Haldane phase is always first order (the energy per site has a kink), in agreement with previous results for $J_{3}=0$ [15]. It is topological: The two phases cannot be distinguished by any local order parameter, but the Haldane phase is topological (supports gapless edge states), whereas the NNN-Haldane phase is not (see sketches in Fig. 1).

For small $J_{2}$, the transition between the Haldane and the dimerized phases is in the $\mathrm{SU}(2)_{2} \mathrm{WZW}$ universality class with central charge $c=3 / 2$ from $J_{2}=0, J_{3} \simeq 0.111$ [9] up to (and including at) a critical end point beyond which the transition becomes first order (see below). There is actually a simple argument in favor of a first-order transition in this parameter

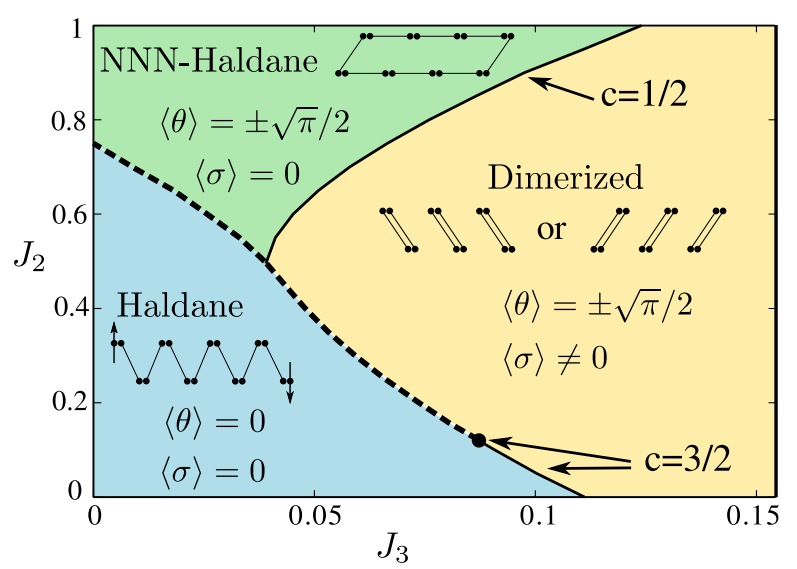

FIG. 1. Phase diagram of the spin-1 chain with next-nearestneighbor coupling $J_{2}$ and three-site interaction $J_{3}$. The transition from the dimerized phase to the Haldane phase is continuous along the solid line with central charge $c=3 / 2$ and first order along the dashed line. The transition from the NNN-Haldane phase to the dimerized phase is a continuous transition in the Ising universality class with central charge $c=1 / 2$. The transition between the Haldane phase and the NNN-Haldane phase is always first order. 

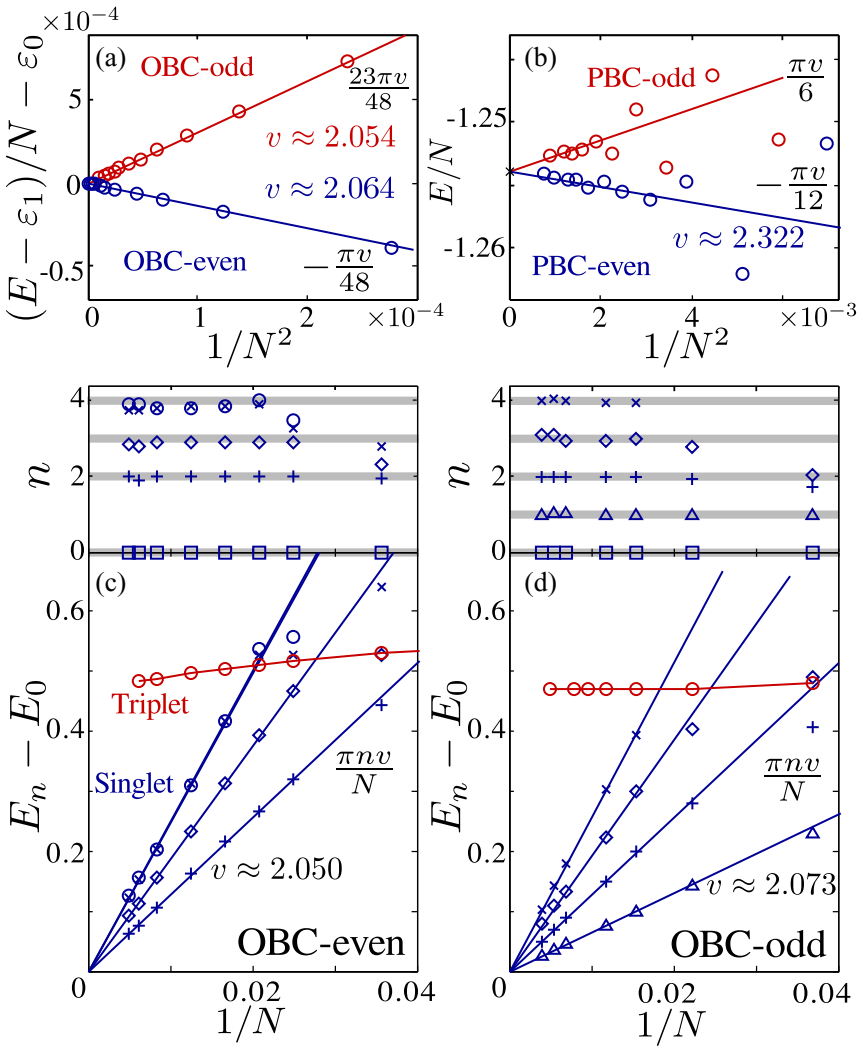

FIG. 2. Ground-state and excitation energies at $J_{2}=0.7$ and $J_{3}=$ 0.058 on the Ising line. (a) Linear scaling of the ground-state energy per site in an open chain with $1 / N^{2}$ after subtracting $\varepsilon_{0}$ and $\varepsilon_{1}$ terms. (b) Linear scaling of the ground-state energy per site with $1 / N^{2}$ in a periodic chain. (c) and (d) Energy gaps in singlet and triplet sectors for $\mathrm{OBC}$ as a function of $1 / N$ for even and odd numbers of sites. The slope of the singlet gap gives values of the velocity. Inset: conformal towers. Gray lines show Ising conformal towers for $I$ ( $N$ even) and $\epsilon$ ( $N$ odd); blue symbols are DMRG data.

range: The fully dimerized state is an exact ground state at $J_{2}=0, J_{3}=1 / 6$ [9], and it remains an exact eigenstate along the line $J_{2}+3 J_{3}=1 / 2$ [16], but it is not a ground state at $J_{2}=1 / 2, J_{3}=0$. So a first-order transition where the dimerization disappears abruptly has to take place. This first-order line connects smoothly at an unusual triple point with the first-order transition between the Haldane and the NNN-Haldane phases [14].

Finally, the transition between the NNN-Haldane and dimerized phases is in the Ising universality class. As shown in Fig. 2, singlet excitations become critical and build a conformal tower whereas the magnetic excitations remain gapped at the transition. Note that we have reached similar conclusions regarding the phase transitions for the spin-1 chain with NNN and biquadratic interactions [14] in partial disagreement with Pixley et al. [17], who in particular reached the conclusion that the transition between the NNN-Haldane and the dimerized phase is first order.

This phase diagram and the nature of the various transitions can be understood using CFT techniques. We begin near the $\mathrm{SU}(2)_{2}$ critical point where the low-energy degrees of freedom of the spin chain can be written in terms of an SU(2) matrix field $g(x, t)$. The staggered component of the spin operators becomes $\vec{S}_{j} \propto(-1)^{j} \operatorname{tr} \vec{\sigma} g(j)$, and the dimerization operator becomes $\vec{S}_{j} \cdot \vec{S}_{j+1} \propto(-1)^{j} \operatorname{tr} g(j)$. The low-energy effective Hamiltonian is that of the $\mathrm{SU}(2)_{2}$ WZW model together with one relevant and one marginal operator,

$$
\mathcal{H}=\mathcal{H}_{W Z W}+\lambda_{1}(\operatorname{tr} g)^{2}+\lambda_{2} \vec{J}_{R} \cdot \vec{J}_{L},
$$

where $\vec{J}_{L / R}$ are the left (right) moving spin currents. The relevant coupling constant $\lambda_{1}$ controls the Haldane to dimerized transition. When $\lambda_{1}<0$, $\langle\operatorname{tr} g\rangle$ becomes nonzero corresponding to dimerization [8]. When $\lambda_{1}>0,\langle\operatorname{tr} g\rangle=0$ corresponds to the Haldane phase. The marginal coupling constant $\lambda_{2}$ renormalizes to zero if it is initially negative. In this regime the Haldane to dimerized transition is second order with the WZW model occurring along the critical line with logarithmic corrections to scaling. These logarithmic corrections vanish at the end of the critical line, where $\lambda_{2}=0$ [18]. When $\lambda_{2}>0$ it renormalizes to large values.

To understand the full phase diagram, it is very useful to use a conformal embedding (also called a coset construction), an exact representation of the $\mathrm{SU}(2)_{2} \mathrm{WZW}$ model as a direct product of a free boson and an Ising model [19]. All operators in the WZW model can be represented as products of free boson and Ising operators. In particular (see the Supplemental Material [20]),

$$
\begin{aligned}
\operatorname{tr} g & \propto \sigma \sin \sqrt{\pi} \theta, \quad(\operatorname{tr} g)^{2} \propto \epsilon-C_{1} \cos \sqrt{4 \pi} \theta, \\
\vec{J}_{L} \cdot \vec{J}_{R} & \propto \epsilon \cos \sqrt{4 \pi} \theta+C_{2} \partial_{x} \phi_{L} \partial_{x} \phi_{R}
\end{aligned}
$$

for constants $C_{1}(>0)$ and $C_{2}$. To see how $\lambda_{1}$ induces the Haldane to dimerized transition, note that a positive $\lambda_{1}$ pins $\theta$ at 0 whereas a negative $\lambda_{1}$ pins it at $\pm \sqrt{\pi} / 2$, leading to $\langle\sin \sqrt{\pi} \theta\rangle \neq 0$. At the same time, a positive coefficient of $\epsilon$ in the Ising Hamiltonian corresponds to the disordered phase whereas a negative coefficient corresponds to the ordered phase with $\langle\sigma\rangle \neq 0$. Thus we obtain, from Eq. (3), $\langle\operatorname{tr} g\rangle \neq 0$ for $\lambda_{1}<0$. Remarkably, in this representation of the WZW model, a second-order transition occurs simultaneously in Ising and boson sectors. The first-order transition for $\lambda_{2}>0$ can be understood intuitively in this representation. A large positive $\lambda_{2}$ favors states with $\langle\epsilon\rangle\langle\cos \sqrt{4 \pi} \theta\rangle<0$. There are then two degenerate gapped states with $\langle\epsilon\rangle<0, \theta$ pinned at 0 corresponding to the Haldane phase or $\langle\epsilon\rangle>0, \theta$ pinned at $\pm \sqrt{\pi} / 2$ corresponding to the dimerized phase. Turning on $\lambda_{1}$ splits the degeneracy, leading to a first-order transition.

So far, we have focused on the vicinity of the WZW critical point. Let us now consider what may happen as we move far from it along the first-order transition line. It is now no longer permissible to only consider the couplings which are relevant at the critical point so the Ising and boson transitions could occur at different places in the phase diagram. For instance, a $\lambda_{3} \cos 3 \sqrt{4 \pi} \theta$ term would favor either $\langle\theta\rangle=0$ or $\langle\theta\rangle= \pm \sqrt{\pi} / 2$ depending on its sign. If $\lambda_{3}$ changed sign along a line in the phase diagram the transition could occur in the boson sector without occurring simultaneously in the Ising sector. This phase with $\langle\theta\rangle= \pm \sqrt{\pi} / 2,\langle\sigma\rangle=0$ corresponds to the NNN-Haldane phase. This can be seen from the presence of gapless $S=1 / 2$ edge excitations when $\langle\theta\rangle=0$ but not when $\langle\theta\rangle= \pm \sqrt{\pi} / 2$. An open boundary favors a dimer ending at the last site. Hence $\langle\operatorname{tr} g(x)\rangle$ becomes nonzero near the 
boundary. Thus $\langle\theta(x)\rangle$ takes the value of $\pm \sqrt{\pi} / 2$ at an open end (see the Supplemental Material [20]), and $\langle\sigma(x)\rangle$ becomes nonzero. However, $\langle\theta(x)\rangle=0$ far from the boundary in the Haldane phase. This rotation of $\theta(x)$ corresponds to an induced magnetization,

$$
\sum_{j} S_{j}^{z}=\int_{0}^{\infty} d x(d \theta / d x) / \sqrt{\pi}= \pm 1 / 2,
$$

near an open boundary at $x=0$ in the Haldane phase. By contrast, there is no induced magnetization in the NNNHaldane phase since $\langle\theta(x)\rangle= \pm \sqrt{\pi} / 2$ in the bulk, so it does not rotate at the boundary. So this phase has no gapless edge modes but also has no dimerization since $\langle\sigma\rangle=0$. Thus, we may identify it with the NNN-Haldane phase. We now see that a third transition can also take place in which $\theta$ remains pinned at $\pm \sqrt{\pi} / 2$ whereas the sign of the $\epsilon$ term in the Hamiltonian changes. This corresponds to an Ising transition from NNN-Haldane to dimerized phases. The gap in the boson sector, at this transition, implies a gap for all magnetic excitations see the Supplemental Material [20]).

Let us now use CFT to extract more precise information about the phase diagram beginning with the Ising transition. As discussed above, an open boundary condition (OBC) favors dimerization, corresponding to a nonzero boundary magnetic field in the Ising model. It then follows from boundary CFT that the magnetization at the critical point decays away from the boundary as [21] $\langle\sigma(x)\rangle \propto 1 / x^{1 / 8}$ since $1 / 8$ is the scaling dimension of $\sigma$. For a finite system of $N$ sites, a conformal transformation gives $\langle\sigma(x)\rangle \propto 1 /[(N / \pi) \sin (\pi x / N)]^{1 / 8}$. On a finite chain, we define the local dimerization as $D(j, N)=$ $\left|\left\langle\vec{S}_{j} \cdot \vec{S}_{j+1}\right\rangle-\left\langle\vec{S}_{j-1} \cdot \vec{S}_{j}\right\rangle\right|$. Identifying the local dimerization with $\sigma$, this leads to $D(j, N) \propto 1 /[N \sin (\pi j / N)]^{1 / 8}$ and in particular to $D(N / 2, N) \propto 1 / N^{1 / 8}$. Plotting $D(N / 2, N)$ versus $N$ on a log-log plot, we determine the Ising critical line by the points where this curve is linear. We find the slope is close to $1 / 8$ along the entire Ising critical line. An example of data on a line crossing the Ising critical line is shown in Fig. 3(a). Along the critical line we also find a good fit of $D(j, N)$ to $1 /[N \sin (\pi j / N)]^{1 / 8}$ as shown in Fig. 3(b).

CFT predicts that all excitation energies, for any conformally invariant boundary conditions, are of the form $(\pi v / N) x_{n}$ where $v$ is the velocity and the dimensionless numbers $x_{n}$ are universal scaling dimensions of operators [22]. Furthermore, the ground-state energy contains a universal term $-\pi v c /(24 N)$ for OBCs and $-\pi v c /(6 N)$ for periodic boundary conditions (PBCs) where $c$ is the central charge. We identify OBCs with $\uparrow, \uparrow$ boundary conditions in the Ising model for $N$ even and $\uparrow, \downarrow$ boundary conditions for $N$ odd where the arrows refer to the directions of the boundary magnetic fields. This follows because OBCs favor the same sign of the dimerization at both ends of the system for $N$ even but opposite signs for $N$ odd. Similarly we identify $\mathrm{PBCs}$ on the spin chain with PBCs on the Ising model for $N$ even but antiperiodic boundary conditions on the Ising model for $N$ odd. We have calculated the ground-state energies in all four cases and the lowest four excited-state energies for $\mathrm{OBCs}$ and both parities of $N$; see Fig. 2. Note that, in stark contrast to the singlet sector, the singlet-triplet gaps in Figs. 2(c) and 2(d) go to nonzero values at $1 / N \rightarrow 0$. These
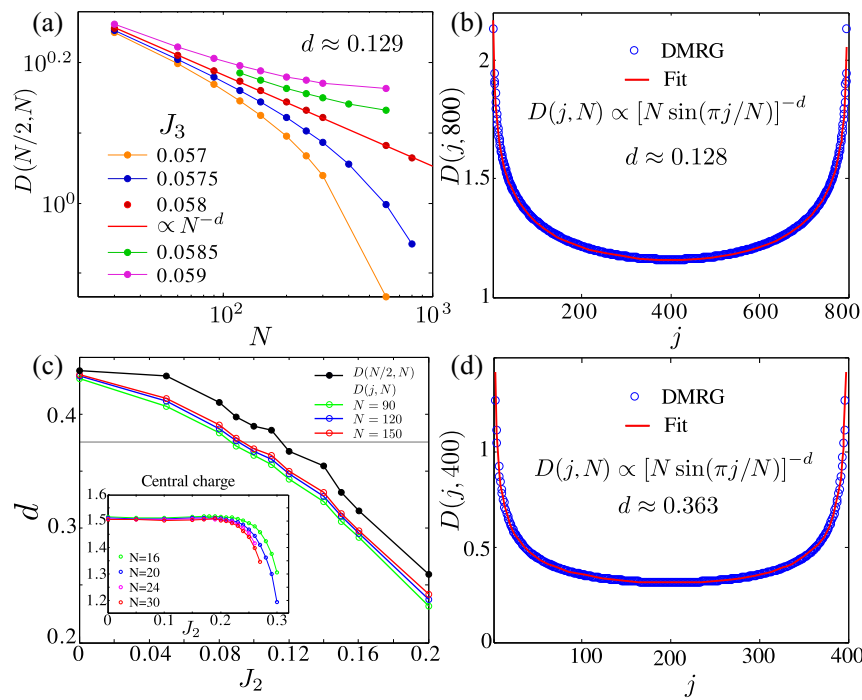

FIG. 3. (a) Log-log plot of the midchain dimerization as a function of the number of sites $N$ for $J_{2}=0.7$ and different values of $J_{3}$. The linear curve corresponds to the Ising critical point and the slope to the critical exponent. This leads to $J_{3 c}=0.058$ and to a slope of 0.129 , in good agreement with the prediction $1 / 8$ for Ising. (b) Site dependence of $D(j, N)$ at the critical point fitted to $1 /[N \sin (\pi j / N)]^{d}$. This determines an exponent $d=0.128$, again close to the Ising prediction of $1 / 8$. (c) Apparent critical exponent along the $\mathrm{SU}(2)_{2}$ critical line as a function of $J_{2}$. Black solid circles: from the slope of the log-log plot $D(N / 2, N)$ as a function of $N$ for the value of $J_{3}$ for which it is linear. Open color circles: from fitting $D(j, N)$ for different sizes at the same points. The dashed line is the theoretical value of the exponent $3 / 8$. The inset: central charge along the critical line as determined from fitting the entanglement entropy of periodic chains with the Calabrese-Cardy formula [23]. (d) $D(j, N)$ at the $\mathrm{SU}(2)_{2}$ critical end point fitted to $1 /[N \sin (\pi j / N)]^{d}$. The exponent is in good agreement with $d=3 / 8$.

data on singlet energies determine ten $x_{n}$ parameters. The nine parameters extracted from OBCs all agree to within 5\% with the CFT predictions for the Ising model (see Table I in the Supplemental Material [20]). The agreement is not as good for PBCs because the sizes accessible to DMRG are much smaller. We plot the excited-state energies in the upper panels of Figs. 2(c) and 2(d). The expected conformal tower structure of excited states is clearly revealed (see the Supplemental Material [20]). Note that the extraction of the central charge from the entanglement entropy for PBCs and OBCs using the Calabrese-Cardy formula [23] is tricky because of the presence of strong oscillations (see the Supplemental Material [20]), but the results are also consistent with $c=1 / 2$.

As stated above, the end point of the WZW SU(2) 2 is characterized by the absence of logarithmic corrections. So this is the only point along the line where the critical exponents can be accurately extracted from finite sizes. For the $\mathrm{SU}(2)_{2}$ model, CFT predicts $D(j, N) \propto 1 /[(N / \pi) \sin (\pi x / N)]^{3 / 8}$. The apparent exponent decreases from $\simeq 0.43$ for $J_{2}=0$ until it reaches $3 / 8$ at $J_{2} \simeq 0.12$ [see Fig. 3(c)]. As a confirmation, we have also extracted the conformal towers at that point for OBCs with $N$ even or odd, and they fit well to the WZW SU(2) 2 prediction (see the Supplemental Material [20]). Along the critical line, the central charge remains equal to $3 / 2$, including at the end 
(a)
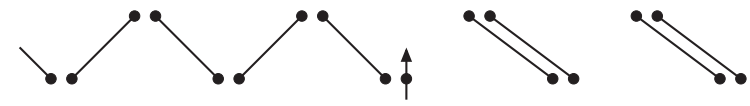

(b)

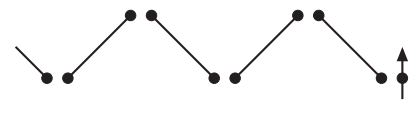

(c)
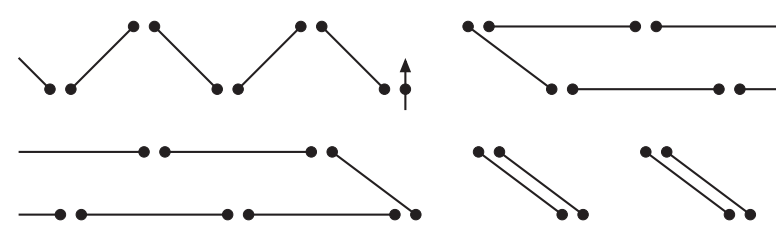

FIG. 4. Sketch of domain walls between (a) the Haldane and the dimerized phases, (b) the Haldane and the NNN-Haldane phases, and (c) the NNN-Haldane and the dimerized phases. A spin-1/2 appears at the domain wall in the first two cases but not in the third one.

point. The fact that it only drops around $J_{2}=0.2$ is presumably a finite-size effect: Since the gap is due to a marginal operator, it increases exponentially slowly above $J_{2}=0.12$ and cannot be seen on small systems.

To summarize, the spontaneous dimerization transition in spin-1 chains can be WZW SU(2) 2 , Ising, or first order depending on the parameters. What is the rationale behind this unexpectedly rich situation? The first observation is that the WZW SU(2) 2 model has a marginal operator, and in such a situation, the transition can be expected to turn first order if its coupling constant changes sign. Regarding the alternative between Ising and WZW SU(2) 2 's, if the transition is continuous, we would like to suggest that it is intimately related to the nature of the domain walls between the phases (see Fig. 4). A domain wall between Haldane and dimerized phases necessarily carries spin- $1 / 2$ because the Haldane phase is topological and has edge states, leading to a transition with magnetic excitations (WZW SU(2) 2 if it is continuous), whereas a domain wall between NNN-Haldane and dimerized phases does not because the NNN-Haldane phase is topologically trivial with no edge states, leading to an Ising transition in the singlet sector with gapped magnetic excitations. These observations are consistent with the fieldtheory approach. At a domain wall between Haldane and dimerized phases $\theta(x)$ rotates by $\pm \sqrt{\pi} / 2$, corresponding to
$S^{z}= \pm 1 / 2$ excitations, whereas at a domain wall between NNN-Haldane and dimerized phases, $\theta$ does not rotate.

The alternative between Ising and $\mathrm{SU}(2)_{2}$ universality classes has been first pointed out by Nersesyan and Tsvelik [24] and Shelton et al. [25] in the related context of spin-1/2 ladders with four-spin interactions using a Majorana fermion representation of the field theory. Calculations on specific models with ring-exchange or frustrated leg coupling have supported this prediction [26-38]. In that respect, the main difference with our model is that, in the model of Nersesyan and Tsvelik [24] and Shelton et al. [25], one goes from Ising to $\mathrm{SU}(2)_{2}$ through a trivial point of decoupled chains $[36,38]$ and central charge $c=2$ with no indication of an end point of the $\mathrm{SU}(2)_{2}$ line followed by a first-order transition, a generic feature of our approach due to the presence of a marginal operator.

Coming back to the role of edge states at the transition, the result summarized in Fig. 4 can easily be extended to ladders to explain the fundamental difference between Ising and $\mathrm{SU}(2)_{2}$ universality classes: spontaneous dimerization transitions between phases which are both topologically trivial (rung singlet and columnar dimer) or nontrivial (Haldane and staggered dimer) can be expected to be generically Ising because edge states are absent or compensate each other, whereas spontaneous dimerization transitions between a topological and a nontopological phase (staggered dimer and rung singlet or Haldane and columnar dimer) must include magnetic excitations because of the edge states and can be expected to be generically $\mathrm{SU}(2)_{2}$ or possibly first order with spin-1/2 solitons. Similar ideas might be extended to transitions between valence-bond solids and dimerized phases in other contexts, possibly in higher dimensions.

F.M. thanks A. Läuchli for very insightful comments on the possible nature of the phase transitions and C. Bazin, P. Lecheminant, and A. Nevidomskyy for useful discussions. This work has been supported by the Swiss National Science Foundation and by NSERC Discovery Grant No. 36318-2009 and CIFAR (I.A.).
[1] F. D. M. Haldane, Phys. Lett. A 93, 464 (1983).

[2] T. Kennedy, J. Phys.: Condens. Matter 2, 5737 (1990).

[3] M. Hagiwara, K. Katsumata, I. Affleck, B. I. Halperin, and J. P. Renard, Phys. Rev. Lett. 65, 3181 (1990).

[4] H. M. Babujian, Nucl. Phys. B 215, 317 (1983).

[5] L. A. Takhtajan, Phys. Lett. A 87, 479 (1982).

[6] I. Affleck, Nucl. Phys. B 265, 409 (1986).

[7] I. Affleck, Phys. Rev. Lett. 56, 746 (1986).

[8] I. Affleck and F. D. M. Haldane, Phys. Rev. B 36, 5291 (1987).

[9] F. Michaud, F. Vernay, S. R. Manmana, and F. Mila, Phys. Rev. Lett. 108, 127202 (2012).

[10] S. R. White, Phys. Rev. Lett. 69, 2863 (1992).

[11] U. Schollwöck, Rev. Mod. Phys. 77, 259 (2005).

[12] S. Östlund and S. Rommer, Phys. Rev. Lett. 75, 3537 (1995).

[13] U. Schollwöck, Ann. Phys. (N.Y.) 326, 96 (2011).

[14] N. Chepiga, I. Affleck, and F. Mila (unpublished).
[15] A. Kolezhuk, R. Roth, and U. Schollwöck, Phys. Rev. Lett. 77, 5142 (1996).

[16] Z.-Y. Wang, S. C. Furuya, M. Nakamura, and R. Komakura, Phys. Rev. B 88, 224419 (2013).

[17] J. H. Pixley, A. Shashi, and A. H. Nevidomskyy, Phys. Rev. B 90, 214426 (2014).

[18] The end of the critical line does not correspond to a $k>2$ WZW model but simply to the point where $k=2 \mathrm{WZW}$ behavior is most clearly observed due to the vanishing of the marginally irrelevant coupling constant there. We disagree in this regard with Ref. [17].

[19] V. Fateev and A. Zamolodchikov, Sov. Phys. JETP 62, 215 (1985).

[20] See Supplemental Material at http://link.aps.org/supplemental/ 10.1103/PhysRevB.93.241108 for further information concerning the field-theory approach, the finite-size spectrum, the 
central charge at the Ising transition, and the convergence of the DMRG energies. This includes Refs. [22,23,39-43].

[21] J. Cardy and D. Llewelyn, Phys. Lett. B 259, 274 (1991).

[22] J. L. Cardy, Nucl. Phys. B 275, 200 (1986).

[23] P. Calabrese and J. Cardy, J. Phys. A: Math. Theor. 42, 504005 (2009).

[24] A. A. Nersesyan and A. M. Tsvelik, Phys. Rev. Lett. 78, 3939 (1997).

[25] D. G. Shelton, A. A. Nersesyan, and A. M. Tsvelik, Phys. Rev. B 53, 8521 (1996).

[26] S. Brehmer, H.-J. Mikeska, M. Müller, N. Nagaosa, and S. Uchida, Phys. Rev. B 60, 329 (1999).

[27] T. S. Nunner, P. Brune, T. Kopp, M. Windt, and M. Grüninger, Phys. Rev. B 66, 180404 (2002).

[28] M. Müller, T. Vekua, and H.-J. Mikeska, Phys. Rev. B 66, 134423 (2002).

[29] K. Hijii and K. Nomura, Phys. Rev. B 65, 104413 (2002).

[30] K. Hijii, S. Qin, and K. Nomura, Phys. Rev. B 68, 134403 (2003).

[31] K. P. Schmidt, H. Monien, and G. S. Uhrig, Phys. Rev. B 67, 184413 (2003).
[32] A. Läuchli, G. Schmid, and M. Troyer, Phys. Rev. B 67, 100409 (2003).

[33] V. Gritsev, B. Normand, and D. Baeriswyl, Phys. Rev. B 69, 094431 (2004).

[34] P. Lecheminant and K. Totsuka, Phys. Rev. B 71, 020407 (2005).

[35] G. Fáth, Ö. Legeza, and J. Sólyom, Phys. Rev. B 63, 134403 (2001).

[36] Y.-J. Wang, Phys. Rev. B 68, 214428 (2003).

[37] A. Lavarélo, G. Roux, and N. Laflorencie, Phys. Rev. B 84, 144407 (2011).

[38] K. Hijii and T. Sakai, Phys. Rev. B 88, 104403 (2013).

[39] J. L. Cardy, Nucl. Phys. B 324, 581 (1989).

[40] S. Capponi, P. Lecheminant, and M. Moliner, Phys. Rev. B 88, 075132 (2013).

[41] A. R. Kolovsky, Phys. Rev. A 77, 063604 (2008).

[42] A. Rocha-Caridi, in Vertex Operators in Mathematics and Physics: Proceedings of a Conference November 10-17, 1983, edited by J. Lepowsky, S. Mandelstam, and I. M. Singer (Springer, Berlin, 1985), pp. 451-473.

[43] I. Affleck and A. W. W. Ludwig, Nucl. Phys. B 352, 849 (1991). 